has extremely high allele loss not only in colorectal carcinomas but also in most adult tumours, including those of breast, brain, lung, bladder, ovaries, cervix, adrenal cortex and bone (see ref. 7). In the small chromosomal region of maximum allele loss at $17 \mathrm{q} 13.1$ is the gene encoding p53. Although initial studies showed no gross rearrangements either in this gene or in its mRNA, Baker et al. have suggested that this is indeed the "colon cancer locus", as two tumours exhibiting allele loss had sustained point mutations in the remaining allele ${ }^{8}$. More tumours have now been investigated by this group and others, and most tumours exhibiting allele loss have missense mutations in the remaining p53 gene ${ }^{7}$. This was the first tumour suppressor gene for colorectal cancer to be isolated, and it has allowed two extremely important questions to be addressed - whether these genes necessarily behave in a recessive manner ${ }^{9}$, and whether allele loss is a second event. The p53 gene has been primarily studied in rodent systems where mutant $\mathrm{p} 53$ gene, in concert with activated ras, transforms primary rat embryo fibroblasts (for review see ref. 5). The mutant genes act dominantly, so that transformation occurs even in the presence of the wild-type allele.

But loss of the wild-type protein has a potentiating effect, which suggests first that the wild-type protein behaves as a tumour suppressor, and second that the mutant and wild-type protein interact, perhaps, as has been suggested, by the oligomerization of the proteins. If the mode of action in the rodent systems holds in human tumour formation, a first mutation, seemingly a point mutation, would confer growth advantage upon the cell, and would be acting in a dominant negative manner rather than in the predicted recessive one. Loss of the wild-type allele could occur later in tumour formation, giving further growth advantage to the cell. If this is the case, it would be expected that those tumours not yet showing allele loss would still have a mutation in one allele. Such tumours have indeed been found, confirming that allele loss is a second event. Mutations in the p53 gene have now also been identified in brain, breast, lung and mesenchymal tumours, showing that this is an extremely important gene in tumour growth ${ }^{7}$

Allele loss on chromosome 5, unlike that on chromosome 17 , seems to be specific to colorectal tumours. In sporadic, or non-inherited, tumours, loss occurs principally in the $5 q 15-q 22$ region, with a peak of loss at 60 per cent (A. H. Wyllie, University Medical School, Edinburgh), strongly indicating that the loss is occurring at the APC gene. Allele loss is also seen in larger sporadic adenomas ${ }^{4}$. Until now, there have been few data on allele loss in carcinomas from APC patients because this material is not commonly

\title{
Finding space for granite intrusions
}

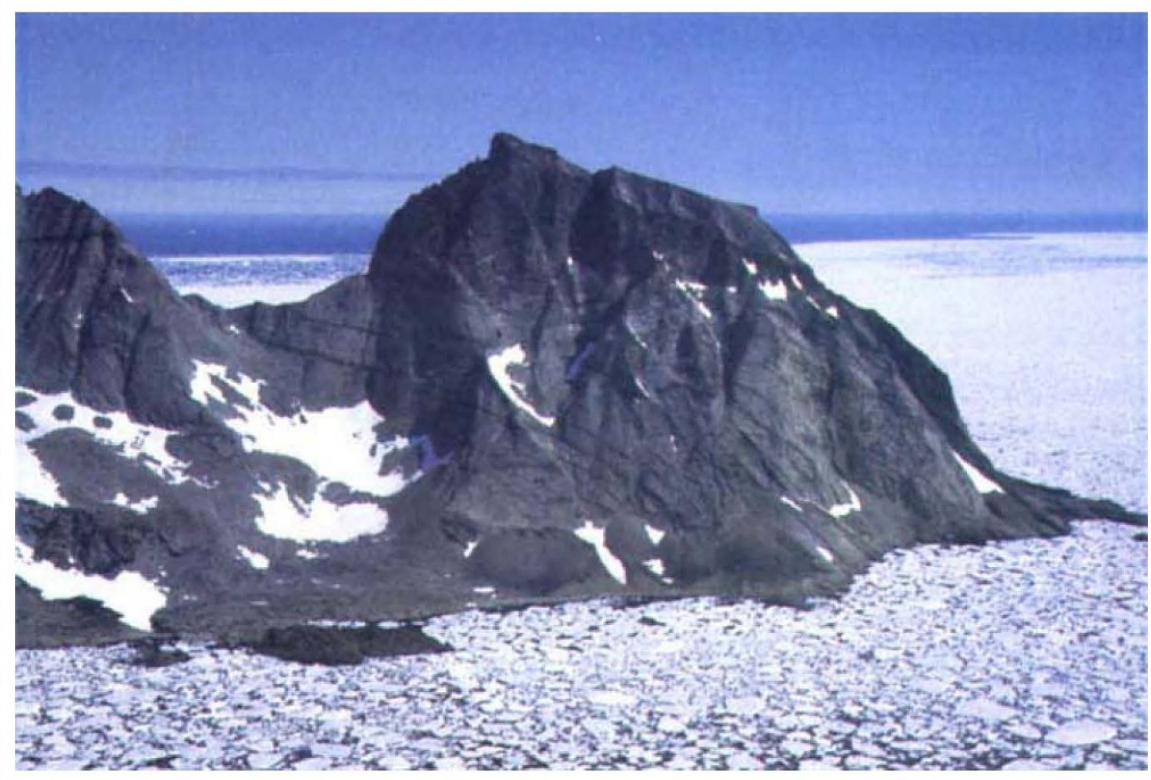

WHERE does the space in the Earth's crust that intrusive granite bodies occupy come from? This problem has long puzzled geologists. Evidence that the intrusions push aside the intruded rocks is usually absent or feeble; flexuring is rarely adequate to provide all the space required. Pieces of host rock partially prised off the roof indicate that blocks could become detached and sink through the rising granite magma. But it is hard to see how the magma could remain molten while a shower of cold blocks sank through it. And anyway, the lack of exposed bases of granite bodies - we usually see the tops or sides - exacerbates the conundrum.

On page 452 of this issue, D.H.W. Hutton and co-workers report their discovery of a remarkably well-exposed granite in Greenland in which both the top and the bottom are exposed. As shown in the photograph, there is a dark band of granite sandwiched between the lighter coloured rocks above and below, towards the top of the mountain. The disposition and structure of the band

available in Western Europe or the United States owing to screening and prophylactic removal of the colon. A Japanese study on a large sample of APC adenomas as well as carcinomas (M. Miyaki, Tokyo Metropolitan Institute of Medical Science) confirmed previous results that allele loss does not occur in the APC adenomas ${ }^{10}$ and dramatically showed that, in APC carcinomas, allele loss from chromosome 5 occurs at the same rate as in the common sporadic carcinomas.

This exciting result neatly ties together a model for the role of the APC gene, along the lines originally predicted by Knudson". In APC patients a single mutation in one allele is inherited, and this mutation is sufficient for adenoma forma- show that the granite was intruded syntectonically, filling the space created by ductile extension along a shear zone created by the separation of crust along an older, gently inclined discontinuity. It is now widely recognized that the pulling apart and extension (by thinning) of crust

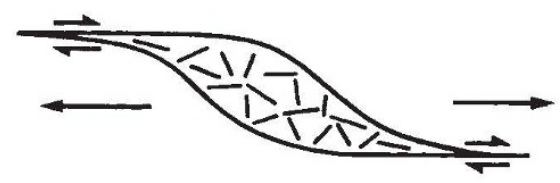

is a common occurrence, so that this discovery probably applies elsewhere. It complements a previous observation, also by Hutton, that spaces open by horizontal movement along vertical faults. Perhaps the largest intrusions, which obviously need the most room, simply filled holes created as the crust was pulled apart.

BERNARD ELGEY LEAKE

Bernard Elgey Leake is in the Department of Geology and Applied Geology, University of Glasgow, Glasgow G12 8QQ, UK.

tion to occur. Again, this gene is clearly not acting recessively at the cellular level. In the progression to carcinoma, loss of the second allele confers full growth advantage. In sporadic cases, the model suggests that a somatic mutation in the APC gene (equivalent to the inherited mutation) occurs early in adenoma formation, and that loss of the second allele, observed later in the progression towards carcinoma formation, is essentially the same event as occurs in the APC tumours. The model cannot be tested fully, however, until this gene is isolated, and the proposed first mutational event can be observed. This same Japanese group was also able to test the model with a carcinoma from an APC individual, in which 